\title{
Exploring Health Care Professionals' Attitudes of Using Social Networking Sites for Health Care: An Empirical Study
}

\author{
Zhao Huang ${ }^{1}$ and NanNan $\mathrm{Gai}^{2}$ \\ ${ }^{1}$ School of Computer Science, ShaanXi Normal University, 710062, P.R. China \\ zhaohuang@snnu.edu.cn \\ ${ }^{2}$ Xi' an No. Five Hospital, 710049, P.R. China \\ nannangai1122@stu.xjtu.edu.cn
}

\begin{abstract}
Evidence from relevant studies show that the use of social networking sites or Web 2.0 portals in health care provide huge potential to transform traditional health care services, generating great collaboration, participation and openness. However, challenges of employing Web 2.0 for health care still exit. This empirical study uses semi-structured interview approach to explore health care professionals' attitudes towards using Web 2.0 portals for health care, especially focusing on specific social networking sites for chronic health care in China. Results present a range of positive attitudes of using the social networking sites to health professionals' clinical practice. Meanwhile, the opportunities and barriers related to use of such social networking sites into clinical practice are presented and discussed.
\end{abstract}

Keywords: E-health, health care services, Web 2.0 portals, Social media, Social networking sites.

\section{Introduction}

Today, for people with a chronic health problem, such as rheumatic diseases or diabetes, the Internet has evolved from being a source for health information presentation (Web 1.0) to being a collaborative resource for connecting with social experiences (Web 2.0) [1]. This change is driven not only by the popularity of Web 2.0, but because user participation. It can strengthen the connections between users (e.g., doctors, nurses and patients) and form a complex network of relation, which in turn enables collaboration, increases user engagement, and promotes service openness [2]. For example, it enables users to access to a peer community where they can discuss their treatment with doctors, nurses and other fellow patients [3]. This means delivers open health information and services, addressing attention to continuous support and problem-based learning for people living with a chronic health problem. On the other hand, the development of medical information on the Internet towards openness and quality control reflects the transformation of health service organizations, where quality surveillance becomes more significant [4], generating 
great cooperation, openness, participation and quality information [5]. Such a new approach echoes the modern management of the chronic health that addresses open education activities and initiatives implemented by health care professionals to help patients with chronic diseases and health conditions to understand their condition and live successfully [6]. For instance, those with rheumatic diseases need to take care of daily physical movement, struggling with undesirable Erythrocyte Sedimentation Rate and the risk of complications, such as interstitial lung disease and primary sjogren syndrome. Long-term assessment of rheumatic treatment addresses the importance of rheumatoid factor changes so as to measure the severity level of rheumatic diseases [21]. Therefore, finding the means to educate and support patients and their families is paramount.

Many studies address the patient perspective, indicating that chronic health care needs improvement of patient information and their accessibility (e.g., [1]). While, some find that effective use of interactive telecare, Internet-based approaches and Web 2.0 applications in health care can promote user access to health services, increase the service delivery as well as information quality, and support patient education (e.g., [7]). Others demonstrate the effects of Web 2.0 on chronic patients' perception, behaviour, attitudes, knowledge, and skills. Although the benefits of using Web 2.0 in health care are recently reviewed (e.g., [8]), only a few studies explore the improvement of quality of life, especially focusing on the health professionals' perspectives. To this end, this paper reported an empirical study that investigates health professionals' attitude towards using Web 2.0 portals, focusing on specific social networking sites for chronic health care in China. Meanwhile, the opportunities and barriers related to use of such social networking sites into clinical practice are presented and discussed.

Accordingly, the paper is presented as follows: Section 2 presents related studies from literature to demonstrate the opportunities and benefits of Web 2.0 applications in health care services. In section 3, an empirical study is designed to explore health care professionals' attitude towards using social networking sites. This allows the detection of opportunities and barriers related to use of social networking sites which are discussed in section 4. Finally, conclusions are drawn and possibilities for future study are recommended in section 5 .

\section{Related Work}

Web 2.0 is a notion where users utilize the web to participate and collaborate using existing web applications and the technologies [9]. Since this concept emerges within the e-health context, the terms Health 2.0 or Medicine 2.0 have been widely used. Eysenbach [2] defines Medicine 2.0 as Web-based services for health care, leveraging Web 2.0 tools, applications and technologies to enable and facilitate social networking, participation, apomediation, openness and collaboration between user groups (including consumers, caregivers, patients, health professional and biomedical researchers). Additionally, Belt et al. [10] use a systematic approach to view the difference between Health 2.0 or Medicine 2.0. The findings indicate that as Health 
2.0 and Medicine 2.0 are still developing areas, there is still no general consensus regarding the definitions of Health 2.0 and Medicine 2.0. These results are also supported by Hughes [11], who state that these concepts seem to have evolved together with the increased use of the definitions and the different parties involved in Healath 2.0 and Medicine 2.0. Hence, no relevant differences exist between Health 2.0 and Medicine 2.0. Although there are no clear definitions of Medicine 2.0 and Health 2.0, or sometime the concepts are exchangeable, it can be argued that Web 2.0 is changing medical practice, transforming traditional health care services and empowering health care users [12].

Jackson et al. [13] investigate interactive computer-assisted technology in diabetes care. The results show that information technology provides an avenue for the rapid and easy dissemination of information to patients and clinicians as well as allowing interactive communication between the patients and their health care provider teams. Moreover, Harno et al. [14] explore diabetes care using an e-health application. They find that the patients start involving e-health because use of e-health in diabetes care for 12 months was able to provide equivalent diabetic control to usual care, and improved cardiovascular risk factors. Such an approach provides the patients with a convenient way to their usual care without physical space and time limitations. Similarly, McMahon et al. [15] assess the effects of web-based care management on glucose and blood pressure control patients. The results present that persistent website users had greater improvement in A1C when compared with intermittent users. Therefore, Web-based care management may be a useful adjunct in the care of patients with poorly controlled diabetes. The above studies suggest that Internet-based interventions have a profound impact on diabetic patients' health care utilization, behaviour, attitude, knowledge and skills.

Existing research investigated the patient views [1], examined e-health service quality [7], and analysed the effects of Web 2.0 on metabolic control with better quality of life [16], while, obtaining limited attention to the health professionals' perspective. It can be argued that without understanding health professionals' attitude toward using Web 2.0 for health care, Health 2.0 or Medicine 2.0 will not reach its full potential. In this vein, this empirical study aims to explore health professionals' attitude toward using Web 2.0 portals, focusing on specific social networking sites for chronic health care in China. By doing so, we hope to contribute to better insights into social networking sites usage in health care by health professionals.

\section{Methodology}

To conduct the study, semi-structured interviews were employed as the research technique with purpose of capturing participants' perception and attitudes of the target social networking sites. The interview was developed based on a set of pre-defined questions. These questions were developed by reviewing relevant evaluation criteria in social media studies in health care (e.g., [17];[19]), and electronic health studies (e.g., [18];[20]). Based on these identified criteria, relevant interview questions were developed and grouped into corresponding question categories. By doing so, we are 
able to provide a step-by-step approach to closely focus on health professionals' attitude towards using target social networking sites.

Two social networking sites (or Web 2.0 portals) were selected as representatives of current social health systems, and targeted to assess health professionals' perception of using them in the study: Huayi.com and Medlive.cn. The former is the one of the most popular social networking sites for health care services in China, involving about two millions health professionals in online health care education, while the latter one is the Chinese largest and most influential medical specialty websites, encouraging social communication and interaction between health professionals and patients. In total, twenty five health care professionals (twenty doctors and five nurses) from three rheumatic diseases units in Xi'an No. Five Hospital took part in this study on a voluntary basis. They all have good knowledge of electronic health systems and experiences using social networking sites. Each participant followed the same interview process consisting of: (1) a free review of the assigned social networking site; (2) answering the interview question. During the interview, the target social networking sites are still available for interviewees to review. Finally, qualitative data were obtained and analyzed.

\section{$4 \quad$ Results and Discussion}

Overall, the results of our study show that there are a range of positive attitudes towards using the target social networking sites to health professionals' clinical practice. Most interviewees were satisfied with the peer community established through the target social networking sites. Using social networking sites provides health professionals with huge opportunities for health information sharing, communication as well as continuous support for problem-based learning. However, problems were given to being familiar with the target social networking sites, showing that interviewees thought they may need to learn more about working with the portals. Furthermore, the findings also identify some major barriers related to utilizing the social networking sites into clinical practice. For example, the process of using social networking sites requires health professionals to offer active contributions from the experience of the present care process in order for them to play a key role in the improvement of care. Nevertheless, most health professionals have had limited computer training either from their prior education or their professional life. Therefore, the requirements of computer training for health professionals become necessary. Furthermore, the results show that health professionals are recently familiar with the rapidly emerging social networking tools on the Internet. In particular, the young health professionals are more likely to accept and use the social networking sites or Web 2.0 portals for health information exchange and communication, but they expect to have more comprehensive understanding or specialized knowledge of using social networking sites or Web 2.0 tools into health care systems. Additionally, since patients' participation and patient generated content, it draws health professionals' greater attention in terms of real-world cases. Several health professionals regard such interactive activities as a valuable source. Hence, a 
need for close collaboration between health care professionals and patients are pointed out when health professional access to the social networking sites.

Moreover, some interviewees described the dissatisfaction with their interaction with the target social networking sites due to the limited time, the lack of the Internet knowledge and insufficient information in the course of the writing process. Although health professionals' experience with the writing process varies, they still feel that a sort of community was built after working with the target social networking sites. Most of them believe that the use of these social networking sites in chronic health could expand health knowledge beyond the clinics, offering more detailed help, support and information for patients. As such, these interviewees are willing to continue working on these social networking sites and expect that more active activities can be involved, as indicated by the following participants' responses:

"I feel confident that such portals become very useful when health professionals and patients work on it......and we are going to use it...... would like to be involved and participate in it in the future."

Regarding the provision of information on the social networking sites, a few interviewees concerned the importance of information quality over the sites, and suggested relevant responsibility from health professionals. They required professional control in order to avoid harmful advice delivery or presenting incorrect references relating to the management of chronic health. A common point made by participants is that access to regularly updated content would encourage patients to take an active role in using the sites as well as learning more about their diseases. The following quotes from the participants' responses indicate their views:

"Site update is used to maintain information and services regularly and keep them up-to-date. A recently updated date is regarded as a key visual cue offering significant reference, which helps patients to determine the quality of information received"; "the last update date indicates regular attention to the site, such a way is beneficial for those who can judge whether information or services obtained are current."

Based on the participants' responses, a significant benefit of the social networking sites is that it enables health professionals to closely communicate and interact with patients and their families. In particular, it is more important for those living with chronic diseases, such as rheumatic diseases, who expect to have continuous support, long-term progress evaluation and problem-based learning. The following quotes indicate the views of the participants:

"I thought the key thing of the Web 2.0 applications in health care is to provide a new way that has the huge potential to change the working environment of the traditional health services to enhance access and patient interaction." 
In addition, some interviewees expressed their concern about the responsibility of acquiring health information. In other words, some patient's parents or family members take more active role in searching for medical advice as well as communicating with health professionals through the social networking sites. To consider the essence of the social networking sites in health care services that emphasize on open education initiatives conducted by health care professionals to help patients with chronic diseases and health conditions to understand their condition and live successfully, there is also a need that involves patients themselves engagement, especially for young patients. After that, several interviewees emphasized that an authorized source of credible health information, for example, references to reliable medical websites, could be very useful support to their work with patients. Such information can provide more detailed health care information, focusing on the specific chronic health problems, as responded in the following: "I like a function on the portals that provides a shared source linking with a number of useful information for the specific chronic health problems. It makes me easier to start working with patients, offering great support, even if I am not familiar with these problems".

\section{Conclusion}

As indicated by recent studies, using social media or Web 2.0 technologies is becoming as a new phenomenon in health care. It brings huge benefits for user groups, such as increased participation and greater collaboration as well as health care organizations, for example accelerating openness health service process and strengthening patient relationships. More importantly, such a way reflects open health service organization perspectives that advocate the modern management of the chronic health, paying more attention to patient continuous support and problembased learning. This empirical study explore health professionals' attitude towards using the social networking sites for chronic health care. The results indicate that health care professionals point to a number of positive attitudes towards employing the social networking sites for health care. Moreover, the findings encourage close collaboration with all user groups when implementing social networking sites for the care of patients with chronic diseases, particularly rheumatic diseases. This study also highlights the need for efforts to educate health professionals in the use of Web publishing, social networking sites, and other Web 2.0 resources. The value of our study lies in the guidance for designers to improve social networking sites for health care, as well as in the help to health service organizations in developing their health care systems.

However, this study is only a small step. There are some important limitations that need to be taken into account when interpreting the findings. First, this study only presents qualitative information about attitudes of health care professionals, which may provide the limited viewpoint of exploration. In order to obtain a more comprehensive research, both qualitative and quantitative data are recommended. Future studies will combine the questionnaire, semi-structure interview and 
observation approach to deeply explore health care professionals' attitudes and task performance when they interact with the social networking sites. In addition, the results show that there is a need to have deep insights into other attitudes of health care team. Such information is beneficial for identifying the specific functions of social networking sites for daily use.

\section{References}

1. Eysenbach, G.: From intermediation to disintermediation and apomediation: new models for consumers to access and assess the credibility of health information in the age of Web 2.0. Stud. Health Technol. Inform. 129(pt. 1), 162-166 (2007)

2. Eysenbach, G.: Medicine 2.0: social networking, collaboration, participation, apomediation, and openness. J. Med. Internet Res. 10(3), 22 (2008)

3. Zrebiec, J.F.: Internet communities: do they improve coping with diabetes? Diabetes Educ. 31(6), 825-828, 830-832, 834, 836 (2005)

4. Nordqvist, C., Hanberger, L., Timpka, T., Nordfeldt, S.: Health Professionals' Attitudes Towards Using a Web 2.0 Portal for Child and Adolescent Diabetes Care: Qualitative Study. J. Med. Internet Res. 11(2), 240-247 (2009)

5. Booth, R.G.: Educating the future eHealth professional nurse. Int. J. Nurs. Educ. Scholarsh. 3, Article 13 (2006)

6. Eysenbach, G.: An Ontology of Quality Initiatives and a Model for Decentralized, Collaborative Quality Management on the (Semantic) World Wide Web. J. Med. Internet Res. 3(4), 34 (2001)

7. Kerr, C., Murray, E., Stevenson, F., Gore, C., Nazareth, I.: Internet interventions for longterm conditions: patient and caregiver quality criteria. J. Med. Internet Res. 8(3), 13 (2006)

8. Eysenbach, G., Köhler, C., Yihune, G., Lampe, K., Cross, P., Brickley, D.: A framework for improving the quality of health information on the world-wide-web and bettering public (e-)health: the MedCERTAIN approach. Stud. Health Technol. Inform. 84(pt. 2), 1450-1454 (2001)

9. Wigand, R.T., Benjamin, R.I., Birkland: Web 2.0 and beyond: implications for electronic commerce. Presented at the 10th International Conference on Electronic Commerce (2008)

10. Van De Belt, T.H., Engelen, L.J., Berben, S.A., Schoonhoven, L.: Definition of Health 2.0 and Medicine 2.0: A Systematic Review. J. Med. Internet Res. 12(2), 18 (2010)

11. Hughes, B., Joshi, I., Wareham, J.: Health 2.0 and Medicine 2.0: Tensions and Controversies in the Field. J. Med. Internet Res. 10(3), 23 (2008)

12. Juzwishin, D.W.M.: Political, policy and social barriers to health system interoperability: Emerging opportunities of Web 2.0 and 3.0. Healthc. Manage. Forum 22(4), 6-10 (2009)

13. Jackson, C.L., Bolen, S., Brancati, F.L., Batts-Turner, M.L., Gary, T.L.: A systematic review of interactive computer-assisted technology in diabetes care. Interactive information technology in diabetes care. J. Gen. Intern. Med. 21(2), 105-110 (2006)

14. Harno, K., Kauppinen-Mäkelin, R., Syrjäläinen, J.: Managing diabetes care using an integrated regional e-health approach. J. Telemed. Telecare 12(suppl. 1), 13-15 (2006)

15. McMahon, G.T., Gomes, H.E., Hickson Hohne, S., Hu, T.M.-J., Levine, B.A., Conlin, P.R.: Web-based care management in patients with poorly controlled diabetes. Diabetes Care 28(7), 1624-1629 (2005) 
16. Hoey, H., Aanstoot, H.J., Chiarelli, F., Daneman, D., Danne, T., Dorchy, H., Fitzgerald, M., Garandeau, P., Greene, S., Holl, R., Hougaard, P., Kaprio, E., Kocova, M., Lynggaard, H., Martul, P., Matsuura, N., McGee, H.M., Mortensen, H.B., Robertson, K., Schoenle, E., Sovik, O., Swift, P., Tsou, R.M., Vanelli, M., Aman, J.: Good metabolic control is associated with better quality of life in 2,101 adolescents with type 1 diabetes. Diabetes Care 24(11), 1923-1928 (2001)

17. Nordfeldt, S., Johansson, C., Carlsson, E., Hammersjö, J.-A.: Use of the Internet to search for information in type 1 diabetes children and adolescents: a cross-sectional study. Technol. Health Care Off. J. Eur. Soc. Eng. Med. 13(1), 67-74 (2005)

18. Falkman, G., Gustafsson, M., Jontell, M., Torgersson, O.: SOMWeb: A Semantic WebBased System for Supporting Collaboration of Distributed Medical Communities of Practice. J. Med. Internet Res. 10(3), 25 (2008)

19. Eysenbach, G., Diepgen, T.L.: Towards quality management of medical information on the internet: evaluation, labelling, and filtering of information. BMJ 317(7171), 1496-1500 (1998)

20. Eysenbach, G.: Credibility of Health Information and Digital Media: New Perspectives and Implications for Youth. In: Digital Media, Youth, and Credibility. The John D. and Catherine T. MacArthur Foundation Series on Digital Media and Learning (2008)

21. Brummaier, T., Pohanka, E., Studnicka-Benke, A., Pieringer, H.: Using cyclophosphamide in inflammatory rheumatic diseases. Eur. J. Intern. Med. 24(7), 590-596 (2013) 